\title{
Nature manuscript 2005-04-04473B
}

\section{Palindromic assembly of the giant muscle protein titin in the sarcomeric Z-disk}

Peijian Zou ${ }^{1 *}$, Nikos Pinotsis ${ }^{1,2^{*}}$, Stephan Lange ${ }^{3,4}$, Young-Hwa Song ${ }^{1}$, Alexander Popov $^{1}$, Irene Mavridis ${ }^{2}$, Olga M. Mayans ${ }^{1,5}$, Mathias Gautel ${ }^{3}$ and Matthias Wilmanns ${ }^{1 \dagger}$

${ }^{1}$ EMBL-Hamburg c/o DESY, Notkeststrasse 85, D-22603 Hamburg, Germany.

${ }^{2}$ Institute of Physical Chemistry, National Center for Scientific Research "Demokritos”, Aghia Paraskevi 15310, Athens, Greece.

${ }^{3}$ The Randall Division of Cell and Molecular Biophysics and Cardiovascular Division, King's College London, Guy's Campus, SE1 1UL London, United Kingdom.

${ }^{4}$ Institute of Cell Biology, ETH Zurich Hoenggerberg, CH-8093 Zurich, Switzerland.

${ }^{5}$ Biozentrum, University of Basel, Division of Structural Biology, Klingelbergstrasse 70, CH-4056 Basel, Switzerland.

*These authors contributed equally to this work. 
The Z-disk of striated and cardiac muscle sarcomeres is one of the most densely packed cellular structures in eukaryotic cells ${ }^{1}$. It provides the architectural framework to assemble and anchor the largest known muscle filament systems by an extensive network of protein-protein interactions, requiring an extraordinary level of mechanical stability. We have unravelled how the $\mathbf{N}$-terminus of the longest filament component, the giant muscle protein titin, is assembled into an antiparallel (2:1) sandwich complex by the Z-disk ligand telethonin. The pseudo-symmetric structure of telethonin mediates a unique palindromic arrangement of two titin filaments, a type of molecular assembly previously found only in protein-DNA complexes. We have confirmed its unique architecture in vivo by protein complementation assays and in vitro by FRET experiments. The model proposed may provide a molecular paradigm of how major sarcomeric filaments are crosslinked, anchored, and aligned within complex cytoskeletal networks.

The Z-disk of the sarcomere defines the lateral boundary of sarcomeric units within the myocyte cytoskeleton. In higher vertebrates, it anchors and aligns at least three major sarcomeric filament systems, including actin, titin, and nebulin ${ }^{1,2}$. It also harbours a large number of smaller protein components, some of which, including $\alpha$-actinin and telethonin, have been mapped to distinct binding sites at the N-terminus of $\operatorname{titin}^{3-7}$. Their presence, proper sorting, and localization within the Z-disk region is critical for myofibril assembly and maintenance of an intact Z-disk structure ${ }^{8}$. Components of the Z-disk are also involved in signalling processes that may regulate muscle development and degradation, as well as in linking contractile functions of muscle sarcomeres to membrane systems like the sarcoplasmic reticulum or the T-tubules associated with excitation-contraction coupling ${ }^{2,8}$. 
The very N-terminal region of titin comprises a domain topology that has been predicted to consist of two immunoglobulin (IG)-like domains, referred to as $\mathrm{Z} 1$ and $\mathrm{Z2}^{9}$. Colocalization studies, two-hybrid interaction screens, and pull-down assays have demonstrated that they interact with the $\mathrm{N}$-terminal region of telethonin at the Z-disk periphery ${ }^{3,4}$. However, although the interaction with telethonin has been considered as a 'cap' (hence, the alternative name 'T-cap'), or 'bolt', it is not known how titin/telethonin binding affects the overall architecture of myofibrils and their associated functions. The physiological importance of this interaction has been supported by evidence linking mutations in the N-terminal regions of titin, telethonin, and the telethonin binding site of Muscle LIM Protein (MLP), to different familial forms of limb-girdle muscular dystrophy (LGMD), as well as hypertrophic and dilated cardiomyopathy (DCM) ${ }^{10-12}$. These findings, along with complementary data using animal models, suggest the existence of a link between the titin/telethonin/MLP interaction and mechanical stress sensor pathways ${ }^{10}$.

Depending on the specific isoform of titin, 200-700 N-terminal residues of multimeric titin filaments are located within and cross over most of the Z-disk of striated muscle sarcomeres $^{2,3,6}$. However, to date, no data are available on the molecular nature of the titin/titin association. Here, we have determined the crystal structure of its N-terminal region in complex with the titin binding domain of telethonin (Fig. 1, Table 1, Supplement Fig. S1). The latter domain is sufficient to localize telethonin to the Z-disk of cardiac myofibrils (Supplement, Fig. S2). Contrary to previous expectations ${ }^{3}$ and previous structural findings on other IG-like domain containing proteins (see Supplement), our data reveal an antiparallel assembly of two titin molecules mediated by telethonin, suggesting that telethonin plays a key role in titin assembly and Z-disk anchoring. 
In the complex, the two N-terminal IG-like domains of titin, Z1 and Z2, are in an extended conformation and are connected by a short three-residue linker. The two domains exhibit a similar structure (r.m.s.d. $=0.66 \AA$, for all common main chain atoms) and sequence (40 of 98 residues are identical). They are almost equally translated by 48 $\AA$ and rotated by $53^{\circ} / 61^{\circ}$ with respect to each other, generating a superhelical coil arrangement of each titin N-terminus. The second component of the complex, telethonin, forms a unique elongated structure with a central five-stranded antiparallel $\beta$-sheet that is extended by two exposed wing-shaped $\beta$-hairpin motifs (A-B, C-D). The two motifs are related by an approximate two-fold symmetry (rotation of $179.4^{\circ}$ ), generating a nearly perfect palindromic arrangement (Fig. 1A). They can be superimposed with an r.m.s.d. of $0.38 \AA$, and in the corresponding structural alignment, 11 of 19 matching residues are similar or identical (Fig. 3B-D).

The peculiar symmetry of the telethonin structure allows it to mediate titin-titin assembly (Fig. 1B). The four nearly identical titin-telethonin interfaces, I-IV, are formed from two intermolecular antiparallel $\beta$-sheets. Within each interface, the long, invariant $\beta$-strand $G$ of one of two titin IG-like domains interacts with one of the four $\beta$-strands $(A, B, C, D)$ of the two wing-like hairpins from telethonin. Hence, each telethonin hairpin (A-B, C-D) provides the core for one of the two (3-2-3)-stranded, antiparallel titin/telethonin/titin $\beta$ sheets. The two $\beta$-sheets are separated by the telethonin core $\beta$-sheet that is flanked by the two short Z1-Z2 linkers of each sandwiching titin molecule. Taken together, the data on the titin/telethonin complex provide a novel concept exemplifying how IG-like domain-containing proteins may act as receptors for protein ligands, such as telethonin. In contrast to other IG-like receptors ${ }^{13,14}$, the protein-protein interface of the titin/telethonin sandwich complex is formed by main chain-mediated intermolecular $\beta$ sheet interactions. In terms of general principles of complex formation of biological 
molecules, the titin/telethonin complex reveals an unprecedented analogy to palindromic or pseudo-palindromic protein-DNA complexes ${ }^{15}$. Future data are needed to determine whether this type of palindromic complex is unique to the titin/telethonin interaction or whether it establishes a novel principle of protein-protein interactions. The extensive network of interactions indicates that the $\mathrm{N}$-terminus of titin, upon complex formation with telethonin, provides a rigid anchoring scaffold rather than adding to the molecular elasticity that has been observed in several titin segments ${ }^{2}$. Indeed, such an anchoring structure that is resistant to external mechanical forces seems to be a prerequisite to allow elastic movements of other parts of titin, specifically within the sarcomeric I-band, under active muscle contraction/relaxation conditions, without the danger of uncontrolled disintegration. The bridging structure of telethonin suggests that it is essential for the functional integrity of the titin filament in mature myofibrils.

To validate our structural data, we first designed an in vitro FRET experiment, in which we introduced four site-specific donor/acceptor fluorophore pairs into the two titin molecules to measure residue-residue distances within the complex in solution (Fig. 2C). The FRET distances of all four donor/acceptor pairs well reflect those observed in the crystal structure (Fig. 2D). Since the FRET data can be neither modelled into a putative parallel titin/titin arrangement, nor explained by other stoichiometries, they provide independent and unambiguous evidence for an antiparallel arrangement of the two titin molecules in the titin/telethonin complex in solution.

Subsequently, we carried out two types of fluorescence imaging experiments to validate our structural findings of the antiparallel titin/telethonin complex under in vivo conditions. In the first approach, we used COS cells to test whether the titin/telethonin complex observed structurally can also form under in vivo conditions in the absence of a pre-existing sarcomeric filament system (Figure $\mathbf{2 A , B}$ ). We employed a yellow 
fluorescence protein (YFP) reconstitution assay using two YFP half domains (YN, YC) ${ }^{16}$. Upon transfection of COS cells with constructs resulting in a titin/telethonin complex, fluorescence from reconstituted YFP was detected only for the YN-titin/telethonin/titinYC system in contrast to the YN-titin/telethonin/YC-titin system, thus fitting an antiparallel assembly of two titin molecules only (Fig. 2A). The data were confirmed by immunoblot assays of lysates from transfected COS cells (Fig. 2B).

In order to examine the correct targeting of telethonin to the endogenous titin N-terminus within the sarcomeric Z-disk in vivo, we used neonatal rat cardiac myocytes (NRC) that express all sarcomeric components (Supplement, Figure S2). To allow comparison of titin/telethonin binding under (i) in vitro conditions, (ii) in test cell line (COS cells) without sarcomeres and (iii) in sarcomere containing muscle cells, we introduced several structure-based single residue mutations in telethonin. In a first experiment series, we changed several residues that are involved in the titin/telethonin interface and tested their ability for titin/telethonin complex formation in vitro (Supplement, Figure S3). None of them indicated an abolishment of the interaction, most likely due to the high stability of the complex formation. However, three telethonin variants, in which the local hydrogen bond pattern of one of two the $\beta$-hairpin wings was disrupted by proline mutations, lost their capacity for binding the titin N-terminus in vitro (Supplement Figure S3) and in COS cell YFP complementation assays (Supplement, Figure S4). In accordance with the molecular data, the same mutants were found to be unable to target correctly to the sarcomeric Z-disk when transfected into NRCs (Supplement, Figure S2), indicating abrogation of complex formation with sarcomeric titin. Taken together, the data suggest that the structural integrity of both pseudo-palindromic telethonin wings are critical for titin/telethonin assembly, regardless whether telethonin assembles with the titin Z1Z2 domains only, as shown in COS cells, or via $\mathrm{N}$-terminal titin within the sarcomeric Z-disk of intact myofibrils (Supplement, Table 1). 
Our model implies that there is a two-fold symmetry in the assembly of the N-terminus of titin, in agreement with previous electron microscopy data ${ }^{17}$. Several arguments have been put forth suggesting that titin:actin thin filaments exist in a 2:1 ratio within the Z$\operatorname{disk}^{6,17,18}$. This stoichiometry could reconcile spacing considerations to match the 28/13 symmetry observed in actin thin filaments, allowing orthogonal $\alpha$-actinin cross-links at $195 \AA$ intervals and satisfying the tetragonal lattice symmetry viewed along the filament axis as well as the estimated Z-repeat distances in titin in the order of $120 \AA$ or less ${ }^{18,19}$. An antiparallel titin/titin arrangement may be plausible because of the localization of the Z-links in the Z-disk centre as previously suggested ${ }^{7,18}$. The titin/telethonin complex structure, however, does not provide direct information about the origin of the two titin molecules that may belong to the same sarcomeric unit or to an adjacent sarcomere. The latter scenario, however, would inevitably lead to relative shifts of titin filaments outside the Z-disk areas in the range of hundreds of $\AA$, which would be in conflict with several imaging studies displaying titin as aligned filaments $3,6,20$. Therefore, the only reconcilable scenario depicts the $\mathrm{N}$-termini of two titin strands as being derived from the same sarcomere.

Within the context of the Z-disk, our structure of the titin/telethonin complex provides an unexpected atomic model for the association of titin molecules at their very N-termini, suggesting that telethonin acts as a titin-titin cross-linker (Fig. 3E). Its molecular architecture, along with evidence from binding and imaging data in this contribution and in previous publications ${ }^{3,4}$, indicates that binding is very tight and may even be irreversible in the absence of signals that would weaken or degrade the interaction. The temporal delay in the proper localisation of telethonin and its selective disappearance in neurogenic atrophy ${ }^{21}$ may suggest that telethonin turnover must be regulated, either intrasterically or by yet unknown alterations in the telethonin structure. In support of this, 
there is accumulating evidence indicating that the observed titin/telethonin assembly may interact with other protein components ${ }^{22-25}$ that generally appear to be more mobile than titin and telethonin ${ }^{7,26}$.

The proposed titin-titin linker function of telethonin is analogous to that of the actin-titin linker of a-actinin ${ }^{6}$. However, compared with the $\alpha$-actinin rod structure, the two terminal $\beta$-hairpin wing motifs in telethonin provide a much shorter linker, leading to a sandwichtype rather than to a rod-type linker model. In this complex, even though the shortest distance between the two titin N-termini is only in the range of 4-15 $\AA$, there are no direct specific titin-titin interactions. Thus, in structural terms, the function of telethonin is to tether the two titin N-termini in close proximity to each other, unlike the a-actinin linker that provides a spacer of more than $200 \AA^{6,27}$. By unravelling the molecular basis of telethonin-mediated titin assembly an overall picture is emerging on how the protein networks in the sarcomeric Z-disk may contribute to titin assembly and anchoring via at least two ligands ( $\alpha$-actinin, telethonin). This structure resists the mechanical forces generated in active muscle sarcomeres ${ }^{28}$ and may feed back to the Z-disk stretch sensor machinery ${ }^{7,10}$. Our data explain how some serious hereditary muscle diseases may be associated with the disruption of molecular interactions that connect and anchor sarcomeric filaments in the Z-disk by bridging mediators. 


\section{Methods}

Preparation of the titin/telethonin complex. A titin construct encoding domains Z1 and Z2 (1-196) and several telethonin variants comprising the full-length sequence (1167) or N-terminal titin binding region (1-90) were cloned, expressed and purified as described previously ${ }^{29}$. In telethonin, C8, C15, C38, C57 and C127 (1-167 only) were mutated into serine residues. Production of the seleno-L-methionine (SeMet)-incorporated telethonin is described in the Supplementary information.

Fluorescence imaging by in vivo complementation. Neonatal rat cardiomyocytes were prepared as described previously ${ }^{30}$. For transfection assays, the pCMV-5 plasmid or the pEGFP plasmids (Clontech) were used. Telethonin was cloned bearing an $\mathrm{N}$ terminal T7-tag ${ }^{\circledR}$ sequence (MTGGQQMGR) or C-terminal GFP-tag, as N-terminal GFP tags were found to act as dominant-negative proteins and to disrupt myofibrils. Transfection of cells was carried out 1 day after plating with a standard liposome carrier system (Escort III) according to the manufacturer. 24-48 hours after transfection cells were fixed in 4\% PFA/PBS for 10 minutes and stained with different antibodies as previously described ${ }^{30}$. For the protein complementation experiments, titin(Z1Z2) cDNA was cloned using PCR and fused to either the $\mathrm{N}$ - or C-terminal region of $\mathrm{YN}(1-$ 172) or YC(156-239) of YFP. COS-1 cells were co-transfected with telethonin(1-90)HA or telethonin(91-163)-HA, together with either YN-titin(Z1Z2) and YC-titin(Z1Z2) or YN-titin(Z1Z2) and titin(Z1Z2)-YC. Cells were fixed 2 days after transfection and stained as described previously ${ }^{30}$.

FRET analysis. Four different single cysteine-containing versions of the titin $\mathrm{N}$ terminus (C3, C18, C99, C195) were used for labelling with Alexa488 (donor) and Cy5 (acceptor). For fluorescence measurements, the titin/telethonin complexes of the four 
mutants and wild-type titin(Z1Z2), as a negative reference, were mixed in the following molar ratios: protein/acceptor, 10:1; protein/acceptor, 1:40; protein/donor, 10:1; protein/donor, 1:40; protein/acceptor/donor, 1:50:1. The labelled probes were separated by gel filtration. To determine the concentration of each dye that is bound to the donor/acceptor sample adducts absorbance was measured for $\lambda=230-900 \mathrm{~nm}$. The fluorescence spectra were scanned for $\lambda=500-800 \mathrm{~nm}$, using an excitation wavelength $\lambda_{\text {ex }}=494 \mathrm{~nm}$. All experiments were carried out in the dark. The energy transfer efficiency, E, of FRET was calculated as a function of the donor-acceptor distance $\left(\mathrm{R}_{\mathrm{AD}}\right) ; \mathrm{E}=1 /\left(1+\left(\mathrm{R}_{\mathrm{AD}} / \mathrm{R}_{0}\right)^{6}\right)$, where $\mathrm{R}_{0}$ is a DA-pair specific constant, the Förster radius $\left(\mathrm{R}_{0}=49 \AA\right)$ for the used donor/acceptor pair. Corrected fluorescence spectra were used to determine the quenching effect of the donor emission intensity when an acceptor is present within the FRET distance. The spectrum of the donor-labelled Z1Z2(C195) mutant (Z1Z2-D) has been used as a reference. E-values were directly determined from the fluorescence intensity: $E=1-I_{A D} / I_{D}$, where $I_{A D}$ is the fluorescence intensity at $524 \mathrm{~nm}$ of the acceptor-donor labelled sample and $\mathrm{I}_{\mathrm{D}}$ the fluorescence intensity of Z1Z2-D at $524 \mathrm{~nm}$, respectively.

X-ray structure determination. Crystals of the titin/telethonin (1-90) complex were grown by vapour diffusion, mixing equal volumes of about $15 \mathrm{mg} \mathrm{ml}^{-1}$ protein solution and mother liquid containing 5\% [w/v] PEG 8000 and $100 \mathrm{mM}$ sodium citrate buffer $(\mathrm{pH} 4.45)$. Crystals were first grown in clusters of thin plates and were then used for macroseeding. In the seeding step, $6 \mathrm{mg} \mathrm{ml}^{-1}$ protein solution was mixed with $7.5 \%$ [w/v] PEG 35000, $100 \mathrm{mM}$ sodium citrate buffer ( $\mathrm{pH} 4.45)$, and $200 \mathrm{mM} \mathrm{Mg} \mathrm{SO}_{4}$. Xray data were collected on the tuneable wiggler beamline BW6 (MPG-ASMB/DESY, Hamburg) and beamline X11 (EMBL/DESY, Hamburg). The X-ray structure was determined using experimental phases from a selenomethionine version of the complex. 
Details of X-ray data acquisition, processing, and structure determination are described in the legend of Table 2 of the Supplementary information. 


\section{References}

1. Clark, K. A., McElhinny, A. S., Beckerle, M. C. \& Gregorio, C. C. Striated muscle cytoarchitecture: an intricate web of form and function. Annu Rev Cell Dev Biol 18, 637-706 (2002).

2. Tskhovrebova, L. \& Trinick, J. Titin: properties and family relationships. Nat Rev Mol Cell Biol 4, 679-89 (2003).

3. Gregorio, C. C. et al. The NH2 terminus of titin spans the Z-disc: its interaction with a novel 19-kD ligand (T-cap) is required for sarcomeric integrity. J Cell Biol 143, 1013-27 (1998).

4. Mues, A., van der Ven, P. F., Young, P., Furst, D. O. \& Gautel, M. Two immunoglobulin-like domains of the Z-disc portion of titin interact in a conformation-dependent way with telethonin. FEBS Lett 428, 111-4 (1998).

5. Sorimachi, H. et al. Tissue-specific expression and alpha-actinin binding properties of the Z-disc titin: implications for the nature of vertebrate Z-discs. $J$ Mol Biol 270, 688-95 (1997).

6. Young, P., Ferguson, C., Banuelos, S. \& Gautel, M. Molecular structure of the sarcomeric Z-disk: two types of titin interactions lead to an asymmetrical sorting of alpha-actinin. Embo J 17, 1614-24 (1998).

7. Wang, J., Shaner, N., Mittal, B., Zhou, Q., Chen, J., Sanger, J.M. \& Sanger, J.W. Dynamics of Z-Band Based Proteins in Developing Skeletal Muscle Cells. Cell Motility and the Cytoskeleton, in press (2005). 
8. Pyle, W. G. \& Solaro, R. J. At the crossroads of myocardial signaling: the role of Zdiscs in intracellular signaling and cardiac function. Circ Res 94, 296-305 (2004).

9. Labeit, S. \& Kolmerer, B. Titins: giant proteins in charge of muscle ultrastructure and elasticity. Science 270, 293-6 (1995).

10. Knoll, R. et al. The cardiac mechanical stretch sensor machinery involves a $\mathrm{Z}$ disc complex that is defective in a subset of human dilated cardiomyopathy. Cell 111, 943-55 (2002).

11. Moreira, E. S. et al. Limb-girdle muscular dystrophy type $2 \mathrm{G}$ is caused by mutations in the gene encoding the sarcomeric protein telethonin. Nat Genet 24, 163-6 (2000).

12. Hayashi, T. et al. Tcap gene mutations in hypertrophic cardiomyopathy and dilated cardiomyopathy. J Am Coll Cardiol 44, 2192-201 (2004).

13. Guddat, L. W. et al. Intramolecular signaling upon complexation. Faseb J 9, 101-6 (1995).

14. Ramsland, P. A. \& Farrugia, W. Crystal structures of human antibodies: a detailed and unfinished tapestry of immunoglobulin gene products. $J$ Mol Recognit 15, 24859 (2002).

15. Remenyi, A., Scholer, H. R. \& Wilmanns, M. Combinatorial control of gene expression. Nat Struct Mol Biol 11, 812-5 (2004).

16. Fang, D. \& Kerppola, T. K. Ubiquitin-mediated fluorescence complementation reveals that Jun ubiquitinated by Itch/AIP4 is localized to lysosomes. Proc Natl Acad Sci U S A 101, 14782-7 (2004).

17. Liversage, A. D., Holmes, D., Knight, P. J., Tskhovrebova, L. \& Trinick, J. Titin and the sarcomere symmetry paradox. J Mol Biol 305, 401-9 (2001). 
18. Luther, P. K. \& Squire, J. M. Muscle Z-band ultrastructure: titin Z-repeats and Zband periodicities do not match. J Mol Biol 319, 1157-64 (2002).

19. Atkinson, R. A. et al. Ca2+-independent binding of an EF-hand domain to a novel motif in the alpha-actinin-titin complex. Nat Struct Biol 8, 853-7 (2001).

20. Furst, D. O., Osborn, M., Nave, R. \& Weber, K. The organization of titin filaments in the half-sarcomere revealed by monoclonal antibodies in immunoelectron microscopy: a map of ten nonrepetitive epitopes starting at the $\mathrm{Z}$ line extends close to the M line. J Cell Biol 106, 1563-72 (1988).

21. Schroder, R. et al. Early and selective disappearance of telethonin protein from the sarcomere in neurogenic atrophy. J Muscle Res Cell Motil 22, 259-64 (2001).

22. Kontrogianni-Konstantopoulos, A. \& Bloch, R. J. The hydrophilic domain of small ankyrin-1 interacts with the two N-terminal immunoglobulin domains of titin. $J$ Biol Chem 278, 3985-91 (2003).

23. Faulkner, G., Lanfranchi, G. \& Valle, G. Telethonin and other new proteins of the Zdisc of skeletal muscle. IUBMB Life 51, 275-82 (2001).

24. Frey, N. \& Olson, E. N. Calsarcin-3, a novel skeletal muscle-specific member of the calsarcin family, interacts with multiple Z-disc proteins. J Biol Chem 277, 139984004 (2002).

25. Furukawa, T. et al. Specific interaction of the potassium channel beta-subunit minK with the sarcomeric protein T-cap suggests a T-tubule-myofibril linking system. $J$ Mol Biol 313, 775-84 (2001).

26. Mayans, O. et al. Structural basis for activation of the titin kinase domain during myofibrillogenesis. Nature 395, 863-9 (1998). 
27. Djinovic-Carugo, K., Young, P., Gautel, M. \& Saraste, M. Structure of the alphaactinin rod: molecular basis for cross-linking of actin filaments. Cell 98, 537-46 (1999).

28. Li, H., Linke, W.A., Oberhauser, A.F., Carrion-Vazquez, M., Kerkvliet, J.G., Lu, H., Marszalek, P.E. \& Fernandez, J.M. Reverse engineering of the giant muscle protein titin. Nature 418, 998-1002 (2002).

29. Zou, P. et al. Solution scattering suggests cross-linking function of telethonin in the complex with titin. J Biol Chem 278, 2636-44 (2003).

30. Auerbach, D. et al. Different domains of the M-band protein myomesin are involved in myosin binding and M-band targeting. Mol Biol Cell 10, 1297-308 (1999) 
Supplementary Information is linked to the online version of the paper at www.nature.com/nature.

Acknowledgements. We thank Dieter Fürst for the kind gift of antibodies. Ralf Kühnemuth is thanked for fruitful discussions on the FRET experiments. Martin Forster was temporarily involved in expression and purification tests. Gleb Burenkov is thanked for assistance during data collection at the beamline BW6 at MPG-ASMB/DESY, Hamburg. Eckhard Mandelkow and Martin von Bergen are thanked for making the fluorimeter at MPG-ASMB/DESY, Hamburg, available, and Elisabeth Ehler is gratefully acknowledged for the preparation of neonatal rat cardiomyocyte cultures. N.P. and S.L. were supported by the EU research and training network CAMKIN to M.W. and M.G., respectively. During involvement at the project O.M. was supported by a EU Marie-Curie postdoctoral fellowship.

Author information. Coordinates and structure factors have been deposited in the Protein Data Bank under the accession number 1YA5. Reprints and permissions information is available at npg.nature.com/reprintsandpermissions. The authors declare that they have no competing financial interests. Correspondence and requests for materials should be addressed to M.W. (wilmanns@embl-hamburg.de). 


\section{Table 1 | Refinement statistics}

Resolution $(\AA)$

Rwork/Rfree (\%)

Number of atoms

Protein

Ion

Water

B factors $\left(\AA^{2}\right)$

Protein

Ion

Water

Root mean square deviations

Bond lengths $(\AA)$

Bond angles (degrees)
$15.0-2.45$

23.2 / 26.5

3726

25

179

49.7

51.2

48.6

0.011

1.370 


\section{Figure Captions}

Figure 1: Structure of the palindromic titin/telethonin/titin complex. Colour codes: titin IG-like domain Z1 (residues 1-98), blue; titin IG-like domain Z2, including the Z1Z2 linker (99-196), cyan; telethonin, red (1-59) and green (60-90). A, Ribbon representation. The relative translations $(\mathrm{T})$ and rotations $(\mathrm{R})$ of the two IG-like domains in each titin molecule and the two $\beta$-hairpin wing motifs, formed by $\beta$-strands $A-B$ and C-D from telethonin, are indicated. B, Schematic representation of the $\beta$-sheet structure in the titin/telethonin $(2: 1)$ complex. The four titin-telethonin $\beta$-sheet interfaces are presented within boxes and are numbered. R87 of telethonin, which is linked to DCM, is coloured in yellow ${ }^{10}$. The $\beta$-sheet hydrogen bonds are depicted by lines. For clarity, only those parts of the two IG-like tandem repeats from titin that are involved in interactions with telethonin are shown. The $\beta$-strands involved with the two titin-telethonin-titin $\beta$ sheets are labelled above and below in colours that correspond to the respective molecules. C, Surface presentation of the titin/telethonin/titin complex in two orientations, rotated by $180^{\circ}$ relative to each other.

Figure 2: Evidence for the formation of the palindromic titin/telethonin complex in vivo and in vitro. A, YFP reconstitution experiments in cotransfected COS cells. Upper panel, N-terminal and C-terminal halves of YFP (YN, YC) were fused to opposite termini of the titin tandem IG-like constructs, YN-titin, and titin-YC. Lower panel, YN and YC were fused to the N-terminus of the titin tandem IG-like construct, yielding YN-titin and YC-titin. (a, d), intrinsic YFP fluorescence; (b, e), detection of HA-epitope tagged telethonin (residues 1-90), using a HA-antibody; (c, f), YC detection, using a GFP- 
specific antibody. The asterisk in (c) refers to a COS cell expressing only one of the two split-GFP constructs, based on the absence of intrinsic YFP fluorescence in (a). B, Western blot analysis of whole COS cell extracts, using a HA antibody for telethonin detection (left panel) and a GFP antibody for detection of split-GFP fusion constructs (right panel). COS cells were cotransfected with the HA-tagged titin binding segment of telethonin (1-90; lanes 1-2), the C-terminal segment of telethonin (91-167; lanes 3-4) as well as either YN-titin and YC-titin (lanes 1 and 3) or YN-titin and titin-YC (lanes 2 and 4), respectively. The $80 \mathrm{kDa}$ band corresponding to the titin/telethonin/titin complex, can be only observed in the case of the antiparallel arrangement; it has been detected in western blot assays using a GFP antibody and using an antibody specific for the HA-tag. C, D, FRET distance analysis of four titin residue pairs from the titin/telethonin complex. The observed FRET distances (inlet, panel C) can only be fitted with an antiparallel arrangement of the two titin molecules in the titin/telethonin complex. The site-specific fluorophore labels are indicated at the corresponding positions in the X-ray structure of the complex. The label colours in panel D correspond to those of the fluorescence spectra in panel $\mathrm{C}$, showing the normalized relative fluorescence spectra of each donor/acceptor pair. The spectrum of the donor-labelled titin(C195) mutant has been used as a reference (in black).

Figure 3: Telethonin-mediated assembly and Z-disk anchoring of titin filaments. (A), Ribbon presentation of the structure of telethonin, indicating the residue numbers of the $\beta$-hairpin wing boundaries; colours as in Figure 1; (B) and (C), stick presentations of the two $\beta$-hairpin wings of telethonin, colours as in panel (A), except those that have been mutated into prolines for validation purposes (in pink). (D), sequence representation of the two $\beta$-hairpin wings, indicating palindromic sequence relations. Identical and 
similar residues are coloured in green and blue, respectively. (E), Model outlining the involvement of the titin/telethonin complex in the architecture of the sarcomeric Z-disk. Titin filaments are assembled by a dual Z-disk bridging system, by $\alpha$-actinin rods on a variable number of titin Z-repeats (three bridges are shown), and by telethonin via the Nterminal IG domains Z1 and Z2. Those components for which high resolution structural data are available are indicated by molecular surfaces, while the remaining components are shown schematically. The titin N-terminus/telethonin complex forms a core that interacts with several ligands both inside and outside the sarcomeric Z-disk. 


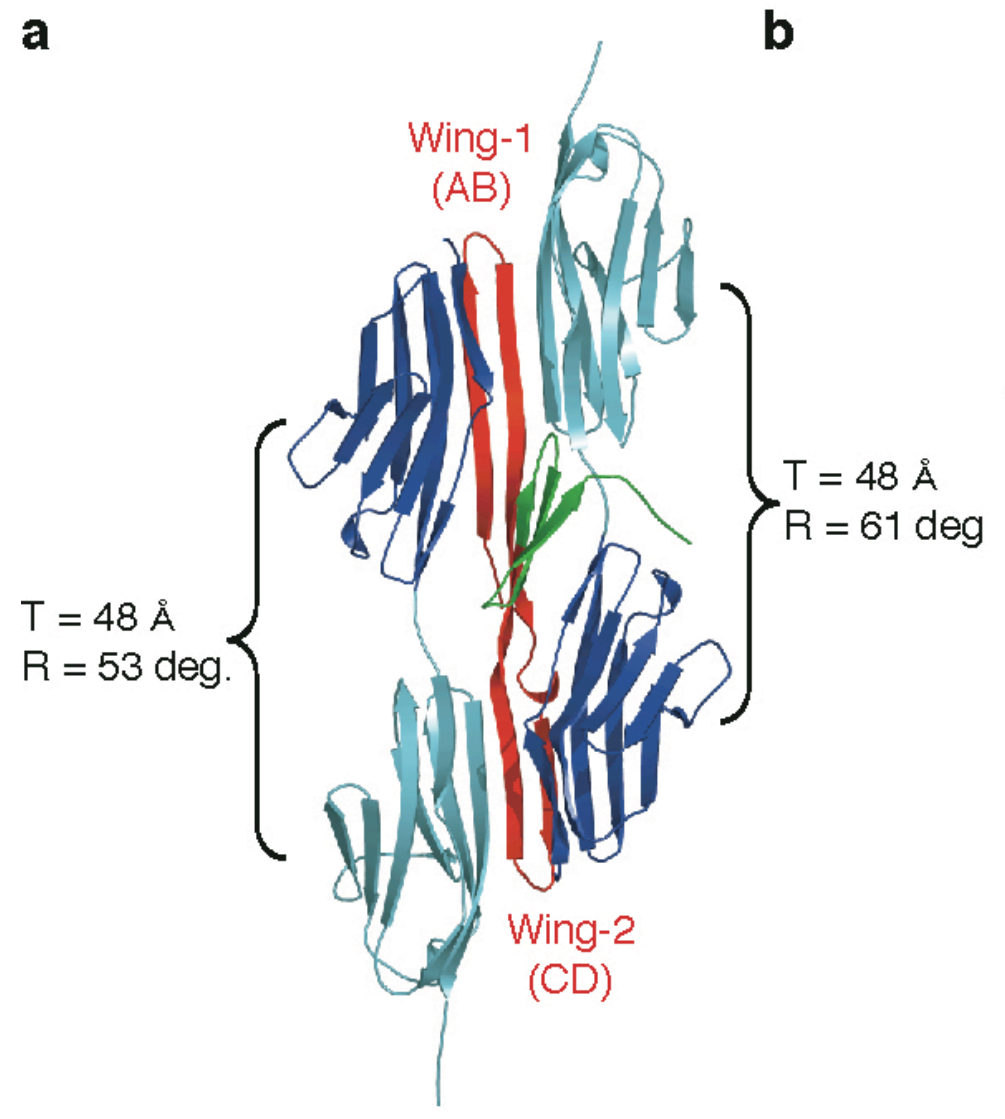

$C-F-G-A-B-G-F-C$

c
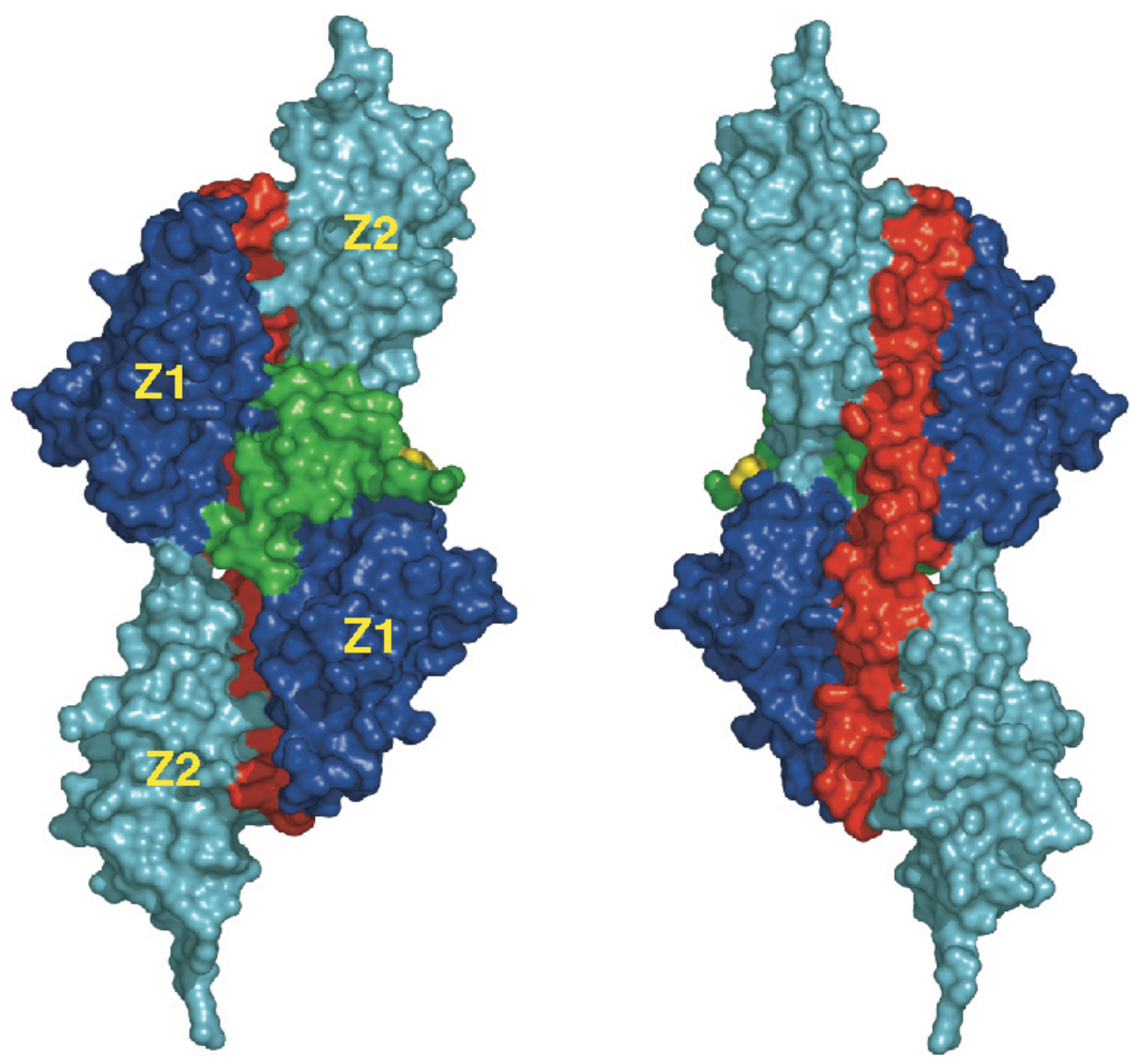
a
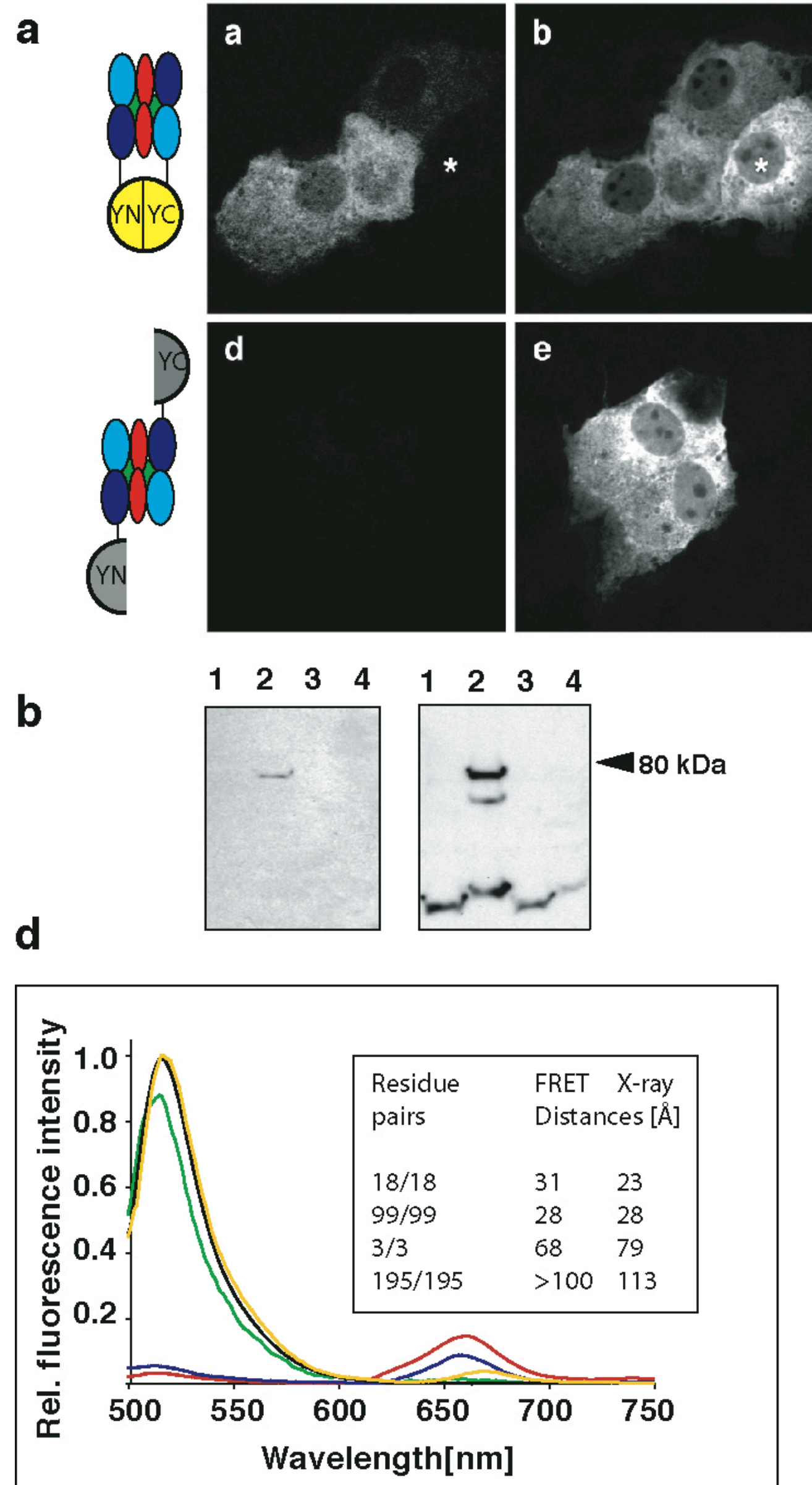
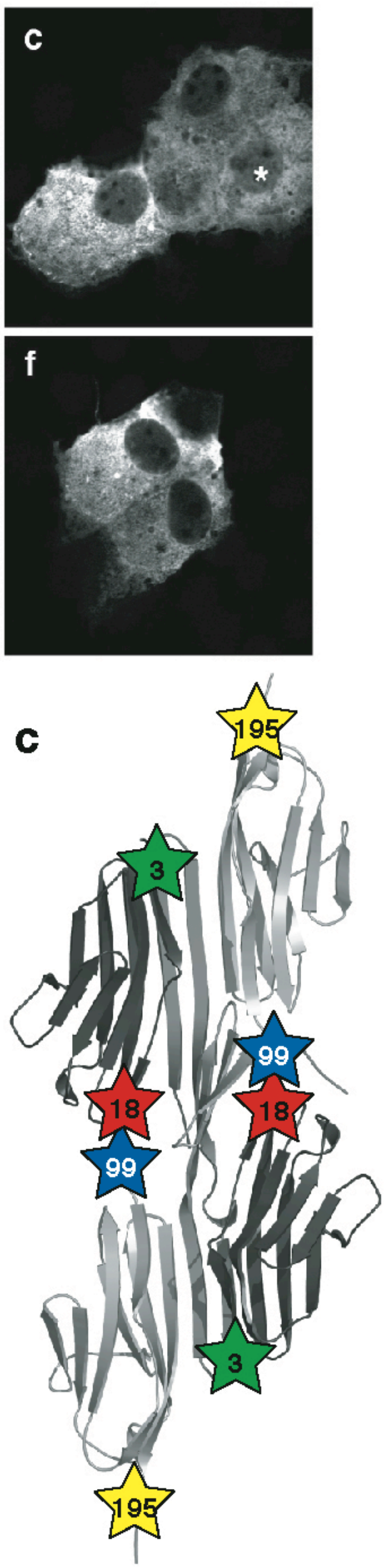
a

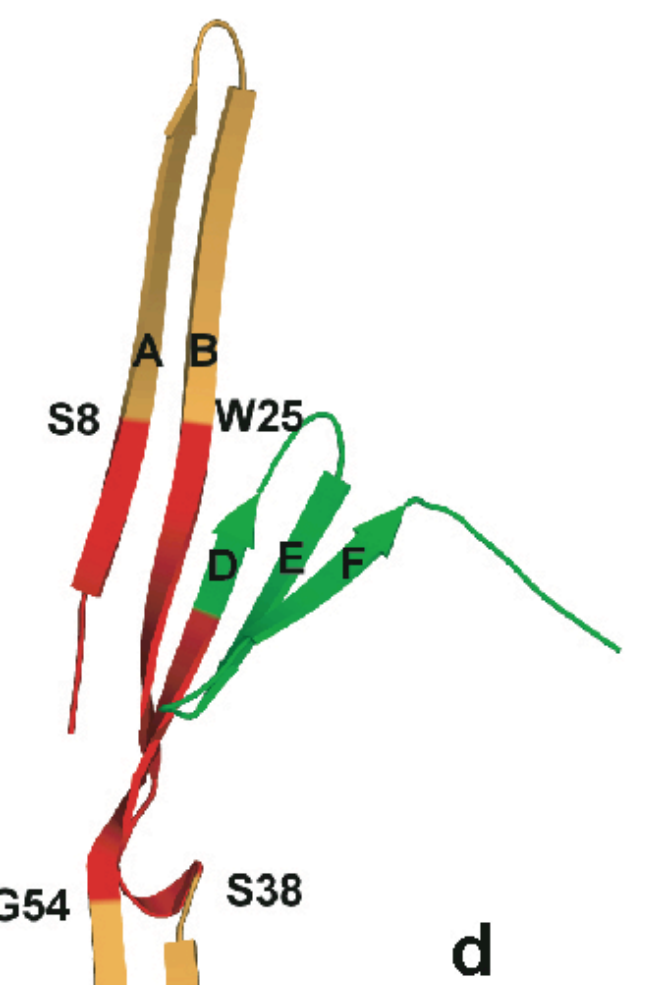

b

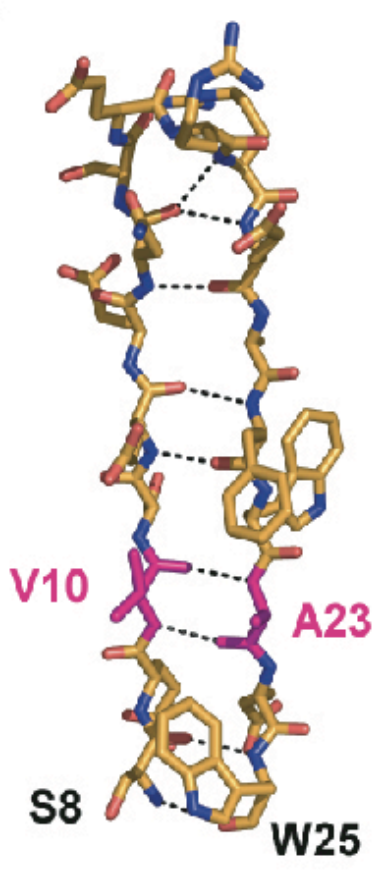

C

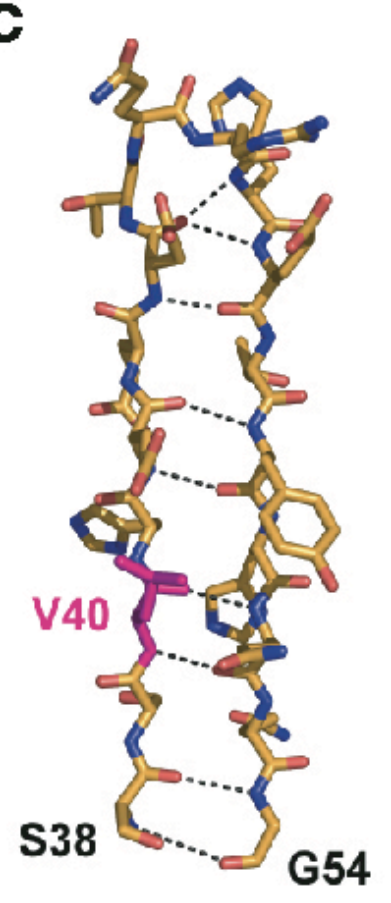

D C

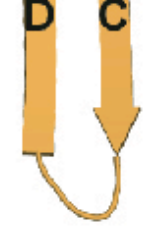

ESNEESVES (08) Wing-1 (AB)

RREAFWAEW (25)

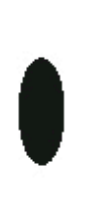

(54) GQQHYTEHR Wing-2 (CD) (38) SSLHEEDTQ<smiles></smiles>

e

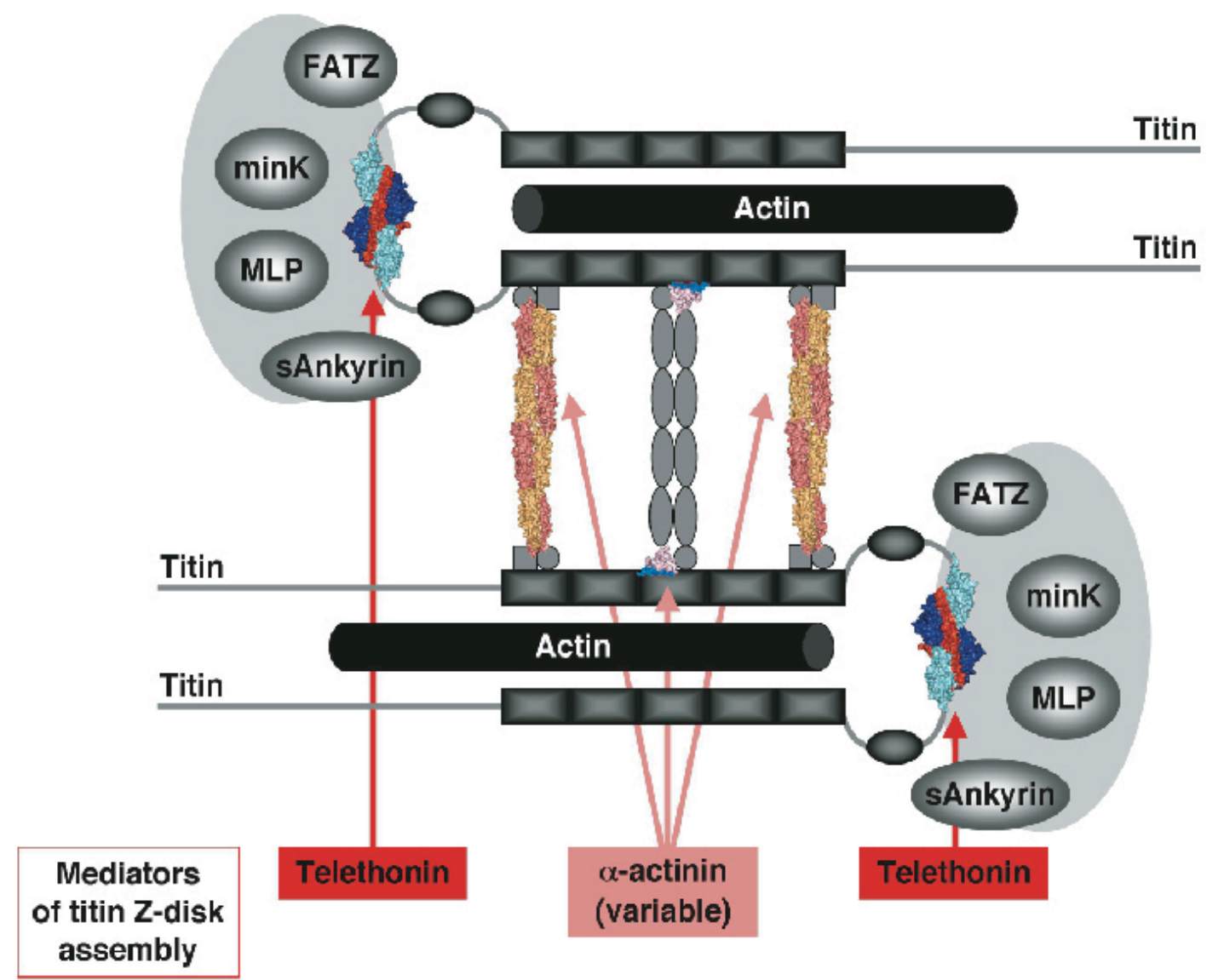

